\title{
Effects of cable fire smoke on electronic boards
}

\author{
LAURENT GAY ${ }^{1}$, ROBERT GRACIA ${ }^{1}$, SYLVERE MONGRUEL ${ }^{1}$, ERIC WIZENNE ${ }^{2}$ \\ ${ }^{1}$ EDF R\&D, 6 quai Watier, 78400 Chatou, France \\ ${ }^{2}$ EDF SEPTEN, 12-14 avenue Dutrievoz, 69100 Villeurbanne, France
}

\begin{abstract}
The consequences of a fire are twofold: an increase in temperature and heat fluxes, and smoke propagation at the source of the fire and in adjacent rooms. These may lead to malfunctions of fire safety electrical equipment in nuclear power plants. A survey of relevant literature has shown that the equipment most affected by smoke particles and gases are electronic boards.

Smoke causes electronic boards to malfunction in four ways: loss of metallic contact due to chemical corrosion, increased short circuiting between the conductors, loss of mechanical contact conductivity, and reduced capacity for movement in electromechanical systems as a result of particle deposits.

These effects can occur over different timescales:

- In the short term, malfunctions that have critical impact in terms of plant safety occur - such as electrical short circuiting and increased contact resistance,

- Mid to longer term, corrosion effects can appear.

A test procedure based on the repeatability of a 20 -minute duration cable fire was set up. Temperature and smoke composition in terms of both gaseous products and soot were controlled. Tests were performed on boards designed to sum test and regulation signals, or to control an analog chain fitted with relays.

Although the behaviour of the equipment may be dependent upon its construction material and equipment layout, no electronic board malfunctions occurred during the 24-hour tests. Furthermore, no malfunctions were detected in the boards when they were retested six months later under normal conditions and without any clean-up following the smoke exposure.
\end{abstract}

KEY WORDS: smoke; reliability; electronic boards; cable fires; malfunction criteria.

\section{INTRODUCTION}

Besides normalization, the behaviour of electrical equipment during a fire - inducing temperature and heat flux increases as well as smoke propagation - will depend on the choice of appropriate material.

The functioning of electrical equipment under conditions of thermal and smoke aggression is an issue of concern for EDF (Electricité de France). The issue of temperature-related effects has been investigated at laboratory-scale by the Research \& Development division at the MILONGA platform via the testing of a sample of "Important for Safety" electrical cabinet devices used in PWR 900 MW French Nuclear Power Plants (NPP). The method developed was based on a definition of the electrical equipment malfunction temperature criteria, following a 'lack of knowledge' statement concerning all but the so-called 'maximum temperature of normal operation' under which continuous function is guaranteed by the equipment manufacturers. For reference, the lowest malfunction temperature was $95^{\circ} \mathrm{C}$ on the board surface and $100^{\circ} \mathrm{C}$ ambient [1].

The consequences of a fire being twofold, this work investigates smoke impact on electronic boards. A survey of the literature has shown that electronic boards are the item of equipment most affected by smoke particles and gases. However, most of the literature concerns forensic studies with relatively little research investigating functioning tests $[2,3,4]$.

To ensure that the cause of any potential malfunctions could not be temperature-induced, the surface temperature of the board was maintained below its defined malfunction criteria.

This work is based on an in-house bench test using an innovative method to burn a solid fuel cable assembly at a quasi-state regime over a period of 20 minutes. The smoke impact was investigated through a performance check on the functioning of the electronic boards, analysis of the fire-generated hot gases and measurement of smoke particles and fire mass loss. 


\section{EFFECTS OF SMOKE}

Smoke provokes electronic board malfunctions in four ways: loss of metallic contact due to chemical corrosion, increased short-circuiting between the conductors, loss of mechanical contact conductivity, and reduced capacity for movement in electromechanical systems (e.g. hard disk) as a result of particle deposits.

These smoke-induced impacts can appear over various timescales:

- Short-term effects include malfunctions such as electrical short circuits and increased contact resistance that are safety critical for the plant; and

- Mid- or long-term effects, such as corrosion.

The types of malfunction obviously depend on the electrical equipment features: in addition to the measurement of amperages and voltages, board functions were also checked.

\section{BOARD SELECTION}

With regard to NPP fire safety rules, the choice was made to perform tests at EDF's in-house designed facility. The tests involved burning PVC cables, smoke from which flowed to a test chamber where the electronic boards were monitored over a 24-hour period. Two electronic boards were selected from the 900 MW NPP equipment (Table 1 \& Fig. 1). These were electricity run and sometimes coupled to thermal components. Table 1 contains a brief outline of the boards with further details of the boards' functions provided in subsequent paragraphs.

Table 1. Electronic board description.

\begin{tabular}{|c|l|l|}
\hline Board & \multicolumn{1}{|c|}{ Function } & \multicolumn{1}{c|}{ Reference } \\
\hline$\# 1$ & Summing of test and regulation signals & Bailey 9020 FE7C12 \\
\hline$\# 2$ & Control of a relay-fitted analog chain & Bailey 9020 FE9H12 \\
\hline
\end{tabular}

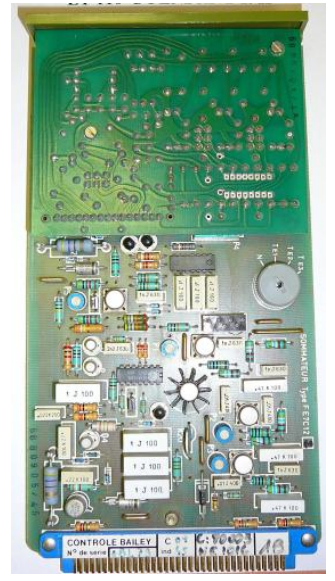

\#1

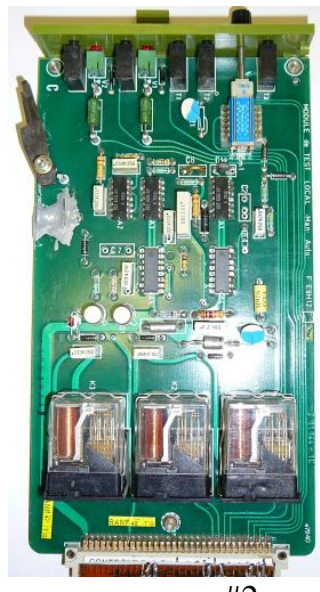

\#2

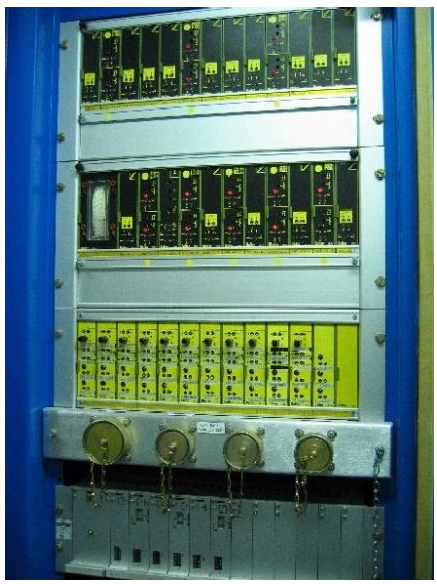

racks

Fig. 1. Boards and racks.

Two series of tests were performed:

(1) In a dynamic regime, in order to check board functioning in an environment in which smoke is circulating;

(2) A series of tests conducted 6 months after smoke exposure, in order to monitor for any long-term impact, which would mainly be due to corrosion. 
A definition of malfunction in terms of smoke impact is provided in the first section of this article. The installation, test procedure, function monitoring, measurement devices and test method are then presented. A protocol validation ensures test repeatability. Results obtained from the dynamic regime and the tests conducted post exposure are discussed in the fourth part of this paper. A conclusion is then drawn regarding smoke impact on electronic boards resulting from a PVC cable fire.

\section{DEFINITION OF MALFUNCTION}

Malfunction is different from failure in the sense that the electronic board may still deliver an output signal and/or continue to operate but will not function accurately. Most of the time, the amperage and voltage outputs are outside their normal working range.

For example, in the case of a board having the function of summing signals such as voltages, malfunction occurs when the output voltage fails under its operating value (above $3.3 \mathrm{~V}$ vs. $3.5 \mathrm{~V}$ ). The following definitions are given with respect to the terms used in this article.

\section{Normal function}

The material operates in accordance with the operating features provided by the manufacturer.

\section{Malfunction}

The material does not comply with the manufacturer-supplied operating feature specifications but is not necessarily out of service or irreversibly damaged. The malfunction operating conditions are defined in a subsequent section of this paper.

\section{Non function}

The material no longer functions and is out of service.

Origin of malfunction according to the electrical equipment features

Malfunction depends on the equipment features, defined as electronically-driven for the boards (Table 2). Malfunction is noted when the output voltage and/or amperage is out of range or when the function is no longer available.

Table 2. Malfunction according to the material type.

\begin{tabular}{|l|c|l|}
\hline Material & Features & \multicolumn{1}{c|}{ Origin of malfunction } \\
\hline Board $\# 1$ & Electronic & Short circuit \\
\hline Board $\# 2$ & $\begin{array}{c}\text { Electronic } \\
\text { Electro-mechanical }\end{array}$ & $\begin{array}{l}\text { Short circuit } \\
\text { Fault in the electro-mechanical relay }\end{array}$ \\
\hline
\end{tabular}

\section{MAFFé TEST BENCH}

The MAFFé (impact of smoke on electronic equipment, in French) test bench (Fig. 2) consists of:

- A metallic furnace with radiant panels for smoke generation $\left(0.8 \times 0.8 \times 0.8 \mathrm{~m}^{3}\right)$,

- An enclosure made of Lexan in which the electronic boards are monitored $\left(1 \times 1 \times 1 \mathrm{~m}^{3}\right)$,

- A duct network and hood for smoke circulation and extraction, and

- Specific measurement devices. 

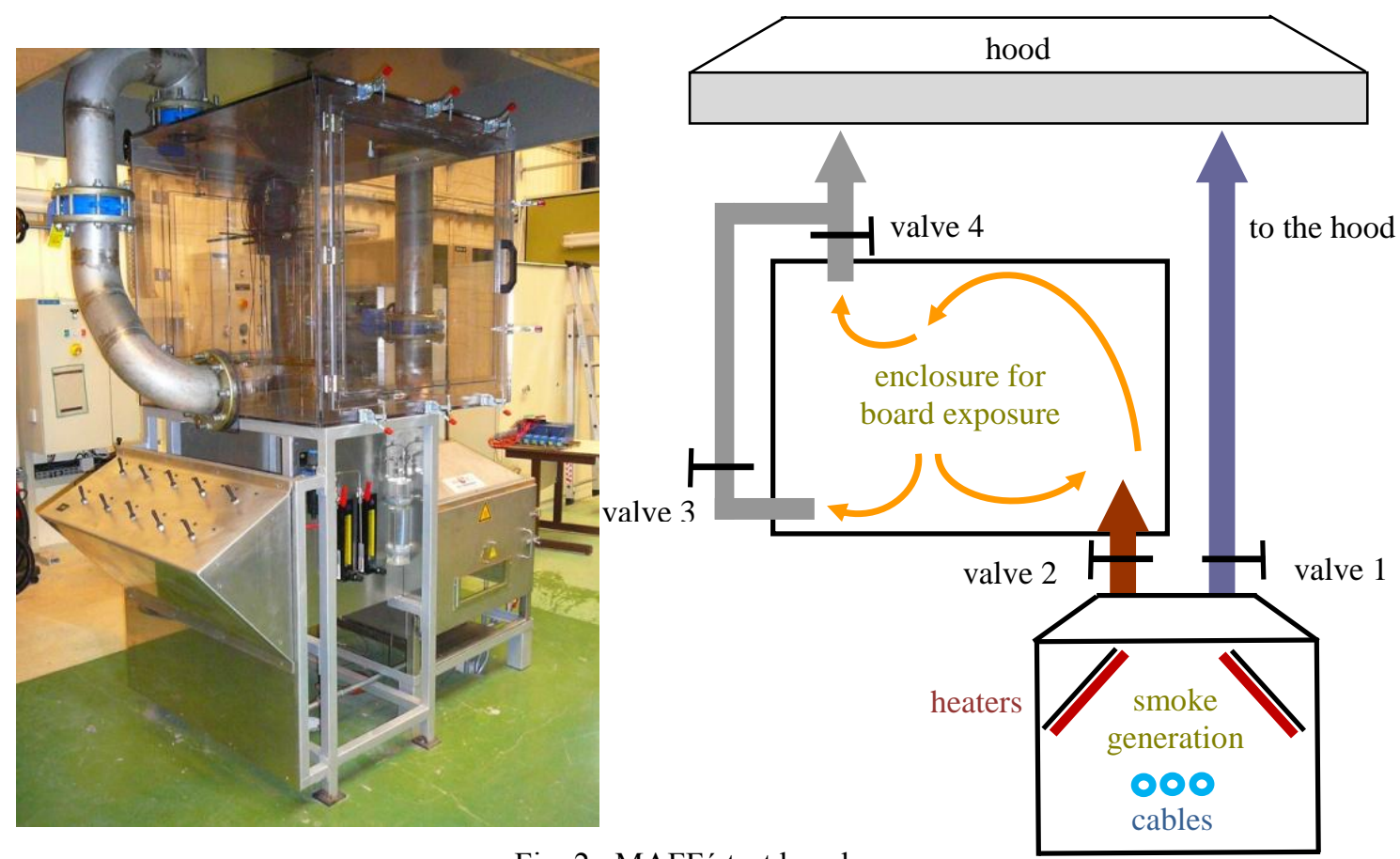

Fig. 2. MAFFé test bench.

\section{Cable sheaths as fuel}

With cables being quantitatively the main potential combustion fuel source in an NPP, a classic PVC low voltage cable was selected as the fuel supply, with controlled burning of its insulation layers (Pirelli 255-3 196104 - lot 1195 - 4 x 1.5 mm² $^{2} 74$ C012.00, Table 3).

Table 3. PVC cable components.

\begin{tabular}{|l|l|}
\hline External sheath & $40 \%$ PVC, $20 \%$ plasticizer, chalk, aluminium, zinc \\
\hline Internal sheath & $60 \%$ PVC, $30 \%$ plasticizer, $10 \%$ mineral fillers \\
\hline Conductor & Copper \\
\hline Plastic filler & N.A \\
\hline Metallic frame & Inox \\
\hline
\end{tabular}

Cables were superimposed in layers to obtain a fire delivering a steady state regime during a 20-minute period. This feature is not trivial for solid fuels and this fire development was the key point during the test set-up (Fig. 3). The total mass of cables is $595 \mathrm{~g} \pm 1 \%$. 

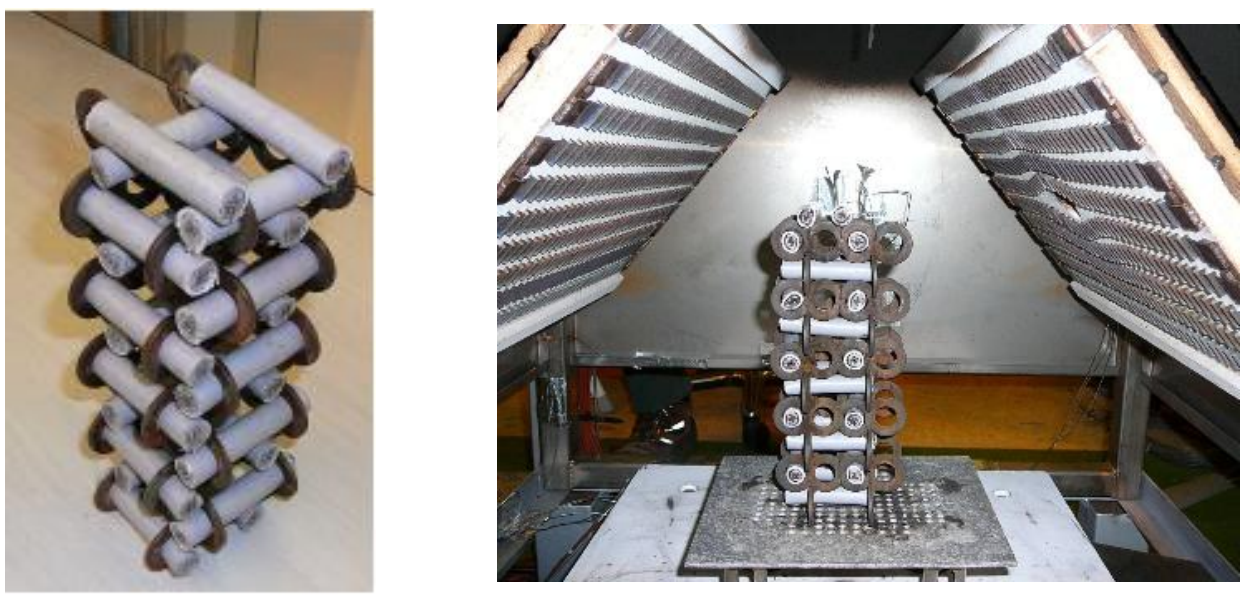

Fig. 3. Cable setup.

\section{Smoke generation in the metallic furnace}

Cables are exposed to a radiant heat flux emitted by electrical panels for a $5 \mathrm{~kW} / \mathrm{m}^{2}$ incident heat flux. The heater regulation is ensured by a control cabinet and a pyrometer.

Cable insulation sheaths attain pyrolysis and their degradation products are ignited with a pilot gas burner. Cable mass loss is measured using a scale balance beneath the cable fire.

Smoke is evacuated through two chimneys, one being connected to the test enclosure and the other to the exit hood for treatment (Fig. 2).

\section{Test enclosure}

The $1 \mathrm{~m}^{3}$ enclosure has one intake connected to the metallic furnace and two outtakes connected to the exit hood. These ducts have valves piloting the enclosure's smoke isolation. The electronic board smoke exposure can thus be controlled sequentially.

The valves have a temperature resistance up to $180^{\circ} \mathrm{C}$ and the Lexan enclosure limitation is $120^{\circ} \mathrm{C}$. In addition, the board enclosure is equipped with measurement probes and devices.

\section{TEST PROCEDURE}

The test procedure is based on a potential cable fire occurrence in an NPP and is set up in order to reproduce the smoke characteristics and fire fighting sequences of this type of event.

Following fire detection, plant safety supervisors have a 20 minute delay in which to isolate the room containing the fire from the ventilation network in case of manual damper activation. For this reason, the MAFFé test bench is equipped with valves to enable gradual exposure of the electronic boards to smoke following the sequential procedure presented below.

\section{Phase \#1: Cable pre-heating, piloted ignition and HRR increase}

Cable surface temperature was the selected criteria for ignition of the pyrolysis gases.

Using radiant panels, the cables are heated from 20 to $270^{\circ} \mathrm{C}$ (internal cable temperature) in approximately 13 minutes $\left(\sim 20^{\circ} \mathrm{C} / \mathrm{min}\right)$. This transient has been set up to obtain a quasi homogeneous temperature inside the cables. At $270^{\circ} \mathrm{C}$, cables released a peak of pyrolysis gases - controlled by a gas analyser - which is then ignited with a propane gas burner [5]. This amount of gas is sufficient to maintain self-sustained combustion under radiant panels.

Phase \#1 corresponds to the detection phase via plant smoke detectors. 


\section{Phase \#2: Self-sustained combustion in a 20min steady-state regime}

This phase corresponds to smoke propagation from the room where the fire is located to an adjacent room; this occurs prior to the time-limit of fire brigade intervention, i.e. 20 minutes after detection. Smoke propagates dynamically in the enclosure.

Valve positions during phases 1 and 2

Valves 2 and 3 are open, valves 1 and 4 closed: pyrolysis followed by the flow of combustion gases into the test enclosure.

\section{Phase \#3: Smoke containment for a period of 2 hours}

This duration phase corresponds to plant equipment fire behaviour, $1 \mathrm{~h} 30$ in the plant used for the purposes of this study or 2 hours for an EPR NPP.

Valve positions during phase \#3

Valves 1,2, $3 \& 4$ are closed: the combustion gases are confined in the enclosure.

\section{Phase \#4: Smoke exit during 10min}

Smoke exits the enclosure via valves 3 and 4 which are opened, valves 1 and 2 remain closed.

\section{Phase \#5: Board functioning observation phase}

The board is no longer surrounded by smoke; however its functioning is monitored for a period of at least 21 hours -eventually up to 45 hours- following exit of the smoke.

Valves 1, 2, $3 \& 4$ are closed.

\section{FUNCTION MONITORING}

Function monitoring was performed throughout the duration of the tests. For boards whose function cannot be continuously recorded, the function was checked regularly. The frequency and level of checking performed is dependent on board components (Table 4).

Table 4. Function checking of the boards.

\begin{tabular}{|c|l|l|}
\hline Board & \multicolumn{1}{|c|}{ Type } & \multicolumn{1}{c|}{ Function checking } \\
\hline$\# 1$ & Summing & $\begin{array}{l}\text { Summing of input currents and checking of the output current } \\
\text { value }\end{array}$ \\
\hline$\# 2$ & Chain control & $\begin{array}{l}\text { Activation of a relay } \\
\text { Output current value checking }\end{array}$ \\
\hline
\end{tabular}

\section{Board \#1 (summing of input currents)}

This module sums 3 input voltages $U_{\text {in } i}$ weighted by a gain $G_{i}$ according to the following formula:

$$
G_{1} \cdot U_{\text {in } 1}+G_{2} \cdot U_{\text {in } 2}+G_{3} \cdot U_{\text {in } 3}=G_{\text {out }} . U_{\text {out }}
$$

The jumper position may vary between [0.1;1]. In this case, it is set to $G_{1}=G_{2}=G_{3}=0.5$ and $G_{\text {out }}=1$.

The offset for the voltage amplifier is set to $0 \mathrm{~V}$. The bias voltage is set to $0 \mathrm{~V}$ with the corresponding jumper varying between 0 and $1.3 \mathrm{~V}$.

Input voltages are set to: $\quad U_{\text {in } 1}=5 \mathrm{~V} \quad U_{\text {in } 2}=U_{\text {in } 3}=1 \mathrm{~V}$

In normal function, the output voltage is equal to $U_{\text {out }}=3.5 \mathrm{~V}$. Thus the malfunction criteria is noted when the output voltage failed to reach $3.3 \mathrm{~V}$ (or eventually exceeded $3.7 \mathrm{~V}$ ). 


\section{Board \#2 (relay)}

Board \#2 is an analog chain control with 3 relays $\mathrm{K} 1, \mathrm{~K} 2$ and $\mathrm{K} 3$. When the board is powered on, all the relays are powered, enabling to pass a voltage.

In the case of malfunction, the power of one, two or three relays is no longer supplied and the indicator signal is no longer provided.

The K1 and K2 relays, when operating simultaneously, are able to cut logic data information from both paths of a voltmetric relay and to deliver contacts for advance warning.

The K3 relay enables to cut off the measurement circuit and substitute it with a reference signal from a test point in the board façade or from the terminal block on the rear face.

Board functioning is controlled by the action of the relays and by monitoring of any voltage passing through the dry contact of each relay.

\section{MEASUREMENTS}

Measurements were specifically directed at board functioning control, enclosure condition monitoring and cable fire characterization.

Temperature is measured with type $\mathrm{T}$ thermocouples with the exception of cable temperature (K thermocouples and pyrometer). For the enclosure temperature, a grid is established to calculate an average temperature. 20 thermocouples are glued to the walls, and on board surfaces with a high-temperature adhesive resin. During the electronic board tests, ambient temperature measurements were taken at a distance of $3 \mathrm{~cm}$ from the board surfaces.

\section{Board instrumentation}

Boards are electrically powered according to the manufacturer's instructions and output signals - current and intensity - representative of board functions are continuously recorded.

\section{Smoke characterization}

Smoke is a heterogeneous mixture of gases and solid particles. Gases may be burnt, not burnt, pyrolysis products or hot air. Gases are determined by their temperature and chemical composition. Solid particles may be soot (mainly constituted of carbon), non-condensable, ambient air particles, etc. These are characterized by their chemical compounds, structures and quantities. Smoke opacity is also an important parameter.

To obtain these measurements, the MAFFé test bench incorporates calibrated continuous gas analysers, a filter bank, a laser and a scale balance to measure cable fire mass loss.

For a given combustion fuel, determinations of the free-burning smoke composition, the fuel mass loss rate (MLR) vs. time, soot mass and smoke extinction coefficient are expected.

\section{Gas analyser}

The gas analyser is composed of three modules: 1) to measure the first $4 \mathrm{CnHm}$ hydrocarbons (Cosma Graphite 55, range 0-9000vpm); 2) to measure the $\mathrm{CO}_{2}$ and $\mathrm{CO}$ (Cosma Cristal 300, $\mathrm{CO}$ range 02000vpm, $\mathrm{CO}_{2}$ range $0-1.5 \%$ ), and 3 ) to measure $\mathrm{O}_{2}$ (Servomex, range $0-21 \%$ ).

\section{Opacity calculation}

Smoke opacity $\mathrm{K}$ is deduced from the depletion of laser-emitted light intensity $\mathrm{I} 0$ crossing an optical medium of a path length $\mathrm{L}$. This signal drop is given by the Lambert-Beer-Bouger law [6]:

$I / I_{o}=e^{-K L}$

$\mathrm{I}$ is the received light intensity. 
For an optical path of length $0.405 \mathrm{~m}$, the opacity is given by:

$K=-2.469 \ln \left(I / I_{o}\right)$

The laser has an emitted light source wavelength of $620 \mathrm{~nm}$ for $0.5 \mathrm{~mW}$ of power as well as an offset reception module.

\section{Bank filter}

The bank filter collects soot in an automated, sealed container. It is located close to the monitored board with 5 samples being collected every 2 minutes. The smoke is extracted over 2 minutes with a vacuum pump and collected on quartz filters (Fig. 4).

The filters are then dehydrated and weighed on a precision scale.

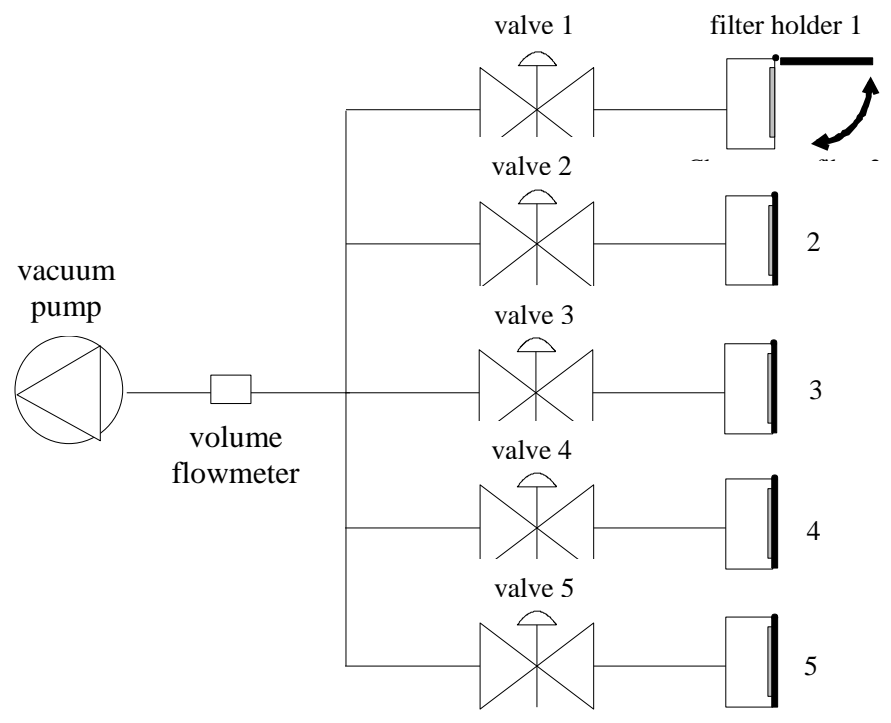

Fig. 4. Bank filter.

\section{Measurement errors}

- Thermocouples, according to tables IEC 584-2:1982 [7]:

Type $\mathrm{T}$ class 1 , uncertainty $\pm 0.5^{\circ} \mathrm{C}$ or $0.004 .|\mathrm{T}|$

Type K class 1 , uncertainty $\pm 1.5^{\circ} \mathrm{C}$ or 0.004 . $|\mathrm{T}|$

- Data acquisition system

IMP board $35952 \mathrm{~A}$ on the range -100 to $400^{\circ} \mathrm{C}$, uncertainty $\pm 0.3^{\circ} \mathrm{C}$

- Scales

Mettler Toledo XS6002S for cable fire, uncertainty $\quad 4 \mathrm{mg}+3 \cdot 10^{-7} . \mathrm{M}$

Mettler Toledo XS603S for soot filter, uncertainty $0.5 \mathrm{mg}+5.10^{-7} . \mathrm{M}$

\section{Test hall conditions}

Hall conditions are defined according to the NF EN 60068 norm [8, 9]:

- Temperature in the range of 15 to $35^{\circ} \mathrm{C}$,

- Relative humidity from 2 to $75 \%$, measured with a HD797T hygrometer $(10-95 \%$ RH range, output $4 \mathrm{~mA}=0 \%, 20 \mathrm{~mA}=100 \% \mathrm{RH}$, temperature $-5-50^{\circ} \mathrm{C}$ ),

- Atmospheric pressure from 86 to $106 \mathrm{kPa}$, measured with a Rosemount pressure gauge (1AP5A22T0285, range 0-1bar, output 4-20mA). 


\section{EXPERIMENTAL PROTOCOL VALIDATION}

Test repeatability is the central parameter for validating the experimental protocol. The boundary conditions imposed on the "important for safety" materials are the smoke characteristics in the enclosure; these are called "the ambient conditions". For a given fire type - in this case a cable fire - three enclosure refill tests were performed to verify that:

- Temperature gradients are reproducible,

- Temperature levels are appropriate to enable distinguishing temperature effects from the smoke impact. Surface temperatures of the boards are below $95^{\circ} \mathrm{C}$,

- Hydrocarbons and smoke opacity evolve along the same order of magnitude from one test to another.

Results provided in Fig. 5 confirm test reproducibility for the PVC cable fire test.
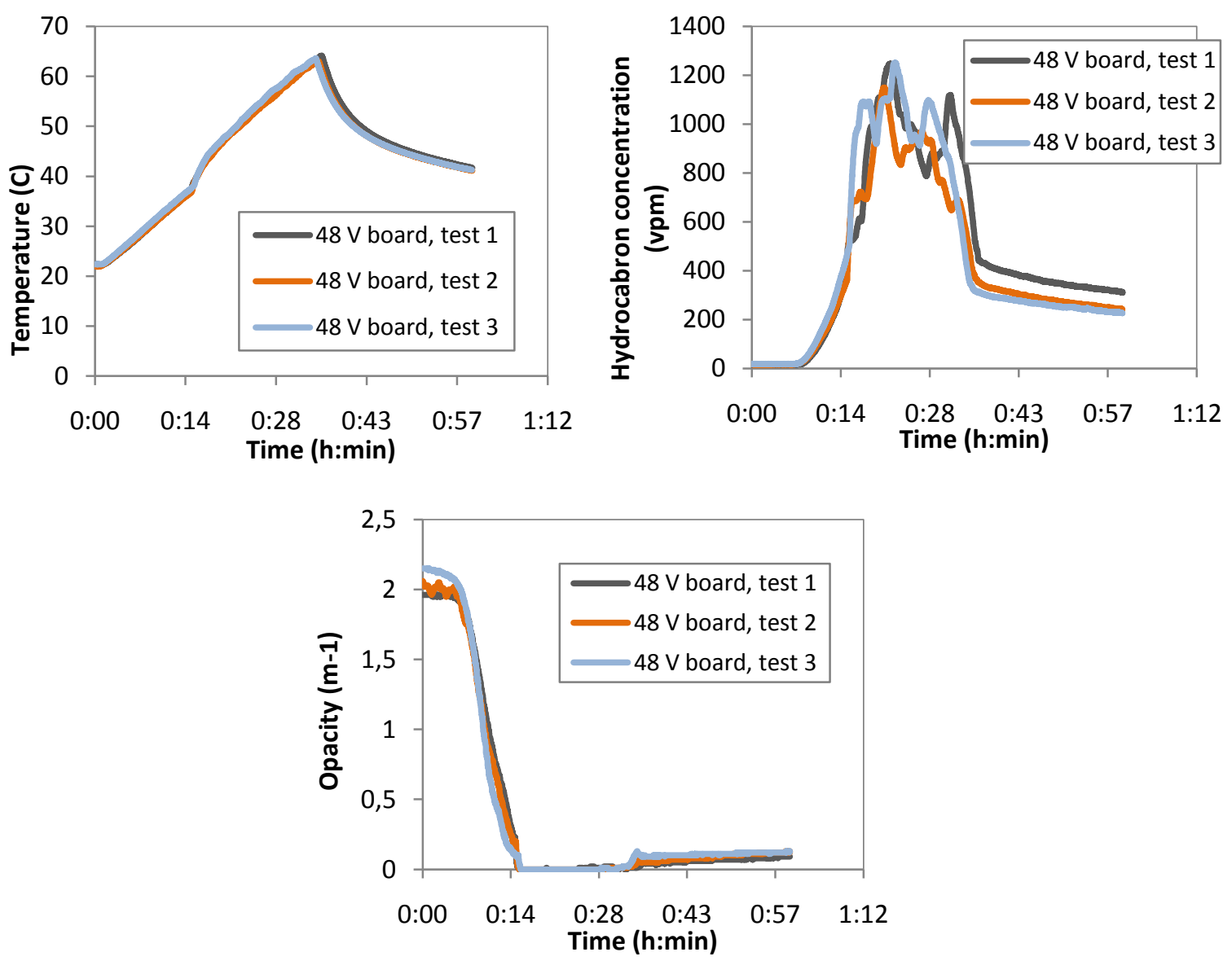

Fig. 5. Board surface temperatures, hydrocarbon concentrations and smoke opacity during validation tests.

\section{RESULTS}

Two tests were conducted on board \#1 and three tests on board \#2. Only one of each series is presented below.

Cables were ignited after about 13 minutes of radiant heat flux exposure. The cable surface temperature reaches a constant value due to the massive release of pyrolysis gases. The internal temperature value falls within the range of $270-280^{\circ} \mathrm{C}$, recognised as being a damage temperature for PVC cables (Fig. 6). 


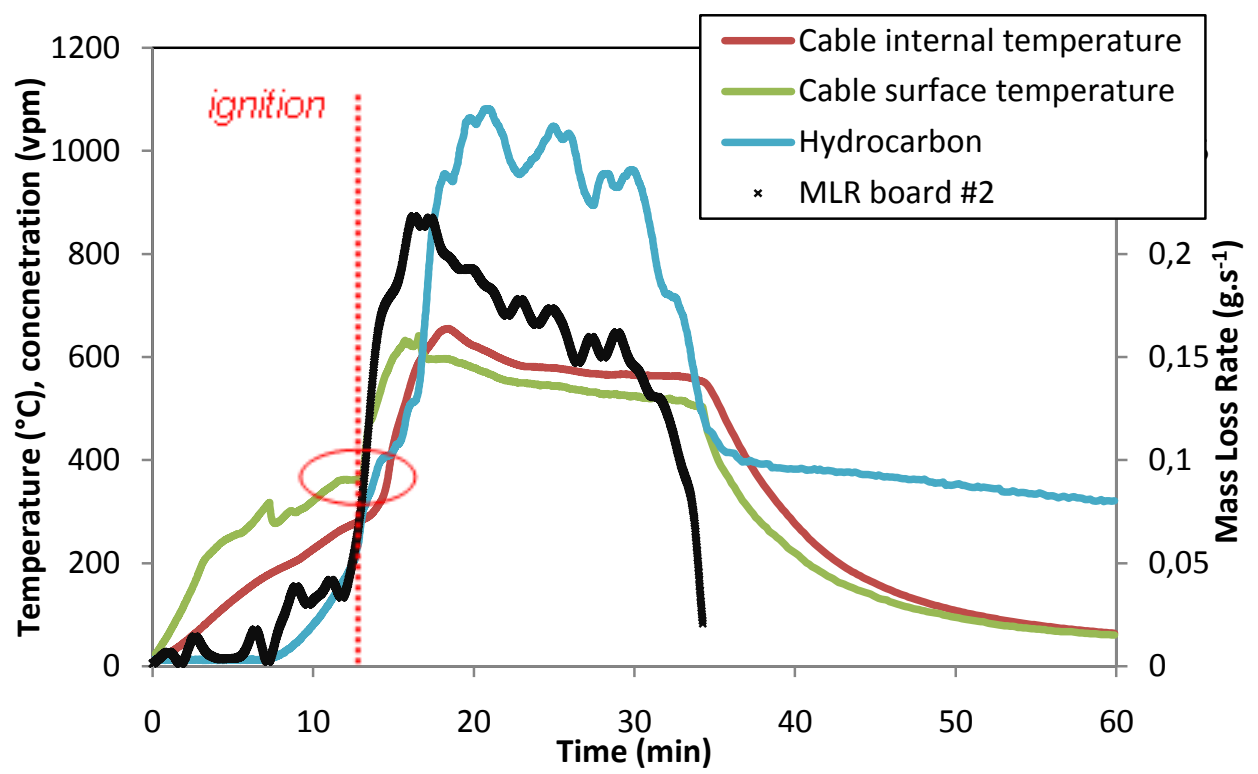

Fig. 6. Cable temperatures, hydrocarbon concentration and cable mass loss rate (board \#2).

Smoke temperature around the board and board surface temperature were the first results of interest. These temperatures evolve according to test phases and should be maintained below the malfunction temperature criteria measured during the previous study [1]. This condition was strictly adhered to for both board tests (Table 5).

Table 5. Smoke and board surface temperatures.

\begin{tabular}{|l|r|r|}
\hline Maximum temperature $\left({ }^{\circ} \mathbf{C}\right)$ & Board \#1 & Board \#2 \\
\hline Smoke & 68.4 & 67.9 \\
\hline Board surface & 56.7 & 56.2 \\
\hline $\begin{array}{l}\text { Thermal malfunction }[1] \\
\text { (board surface) }\end{array}$ & 115 & 101 \\
\hline
\end{tabular}

The in-house procedure works reasonably well, with the MLR attaining a type of steady state regime that is difficult to obtain for solid fuel burning (Fig. 7 and 8). For board \#1, the MLR value is $0.18 \pm 0.03 \mathrm{~g} . \mathrm{s}^{-1}$ for $17 \mathrm{~min}$. 


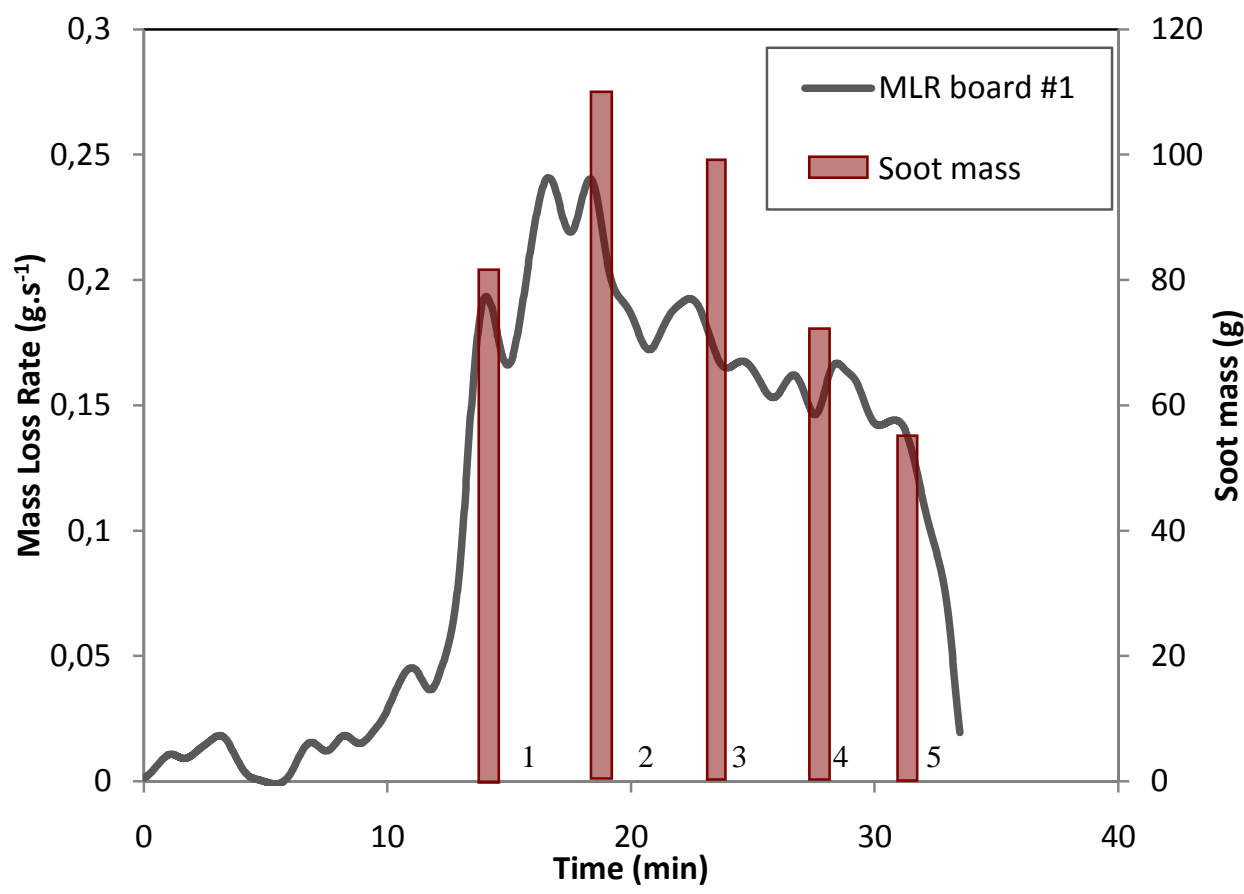

Fig. 7. Mass loss rate and soot production results for board \#1 test.

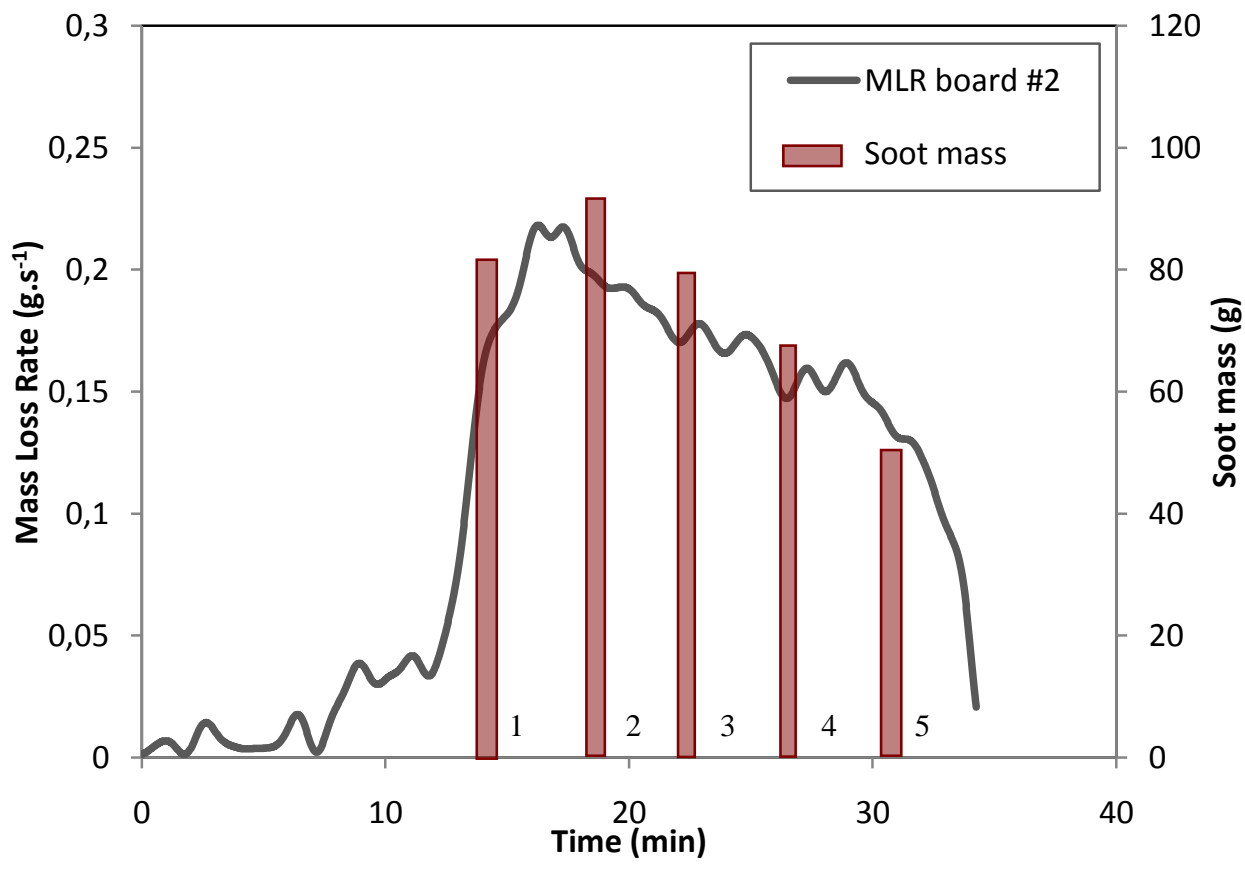

Fig. 8: Mass loss rate and soot production for board \# 2 test.

The soot is collected on filters. Its mass quantity is linked to the cable MLR and the enclosure volumerelated smoke dilution. Five samples are made during the $20 \mathrm{~min}$ fire duration, from $13 \mathrm{~min}$ to $33 \mathrm{~min}$ (Table 6). 
Table 6. Soot mass on filters.

\begin{tabular}{|c|c|c|c|}
\hline Board & Sample & $\begin{array}{c}\text { Sample } \\
\text { duration (s) }\end{array}$ & $\begin{array}{c}\text { Soot mass } \\
(\mathbf{m g})\end{array}$ \\
\hline$\# 1$ & 1 & 119 & 68 \\
& 2 & 121 & 90 \\
& 3 & 121 & 79 \\
& 4 & 122 & 65 \\
$\# 2$ & 5 & 120 & 53 \\
\hline & 1 & 119 & 68 \\
& 2 & 121 & 90 \\
& 3 & 119 & 79 \\
& 4 & 120 & 65 \\
& 5 & 124 & 53 \\
\hline
\end{tabular}

Concentration levels for the first four hydrocarbons are presented in Fig. 9. The graph presented therein demonstrates an obvious relationship between the peak of hydrocarbons and the MLR dynamic. This is equally the case for the smoke opacity as calculated on the basis of measurements of the laser-emitted light extinction (Fig. 10).

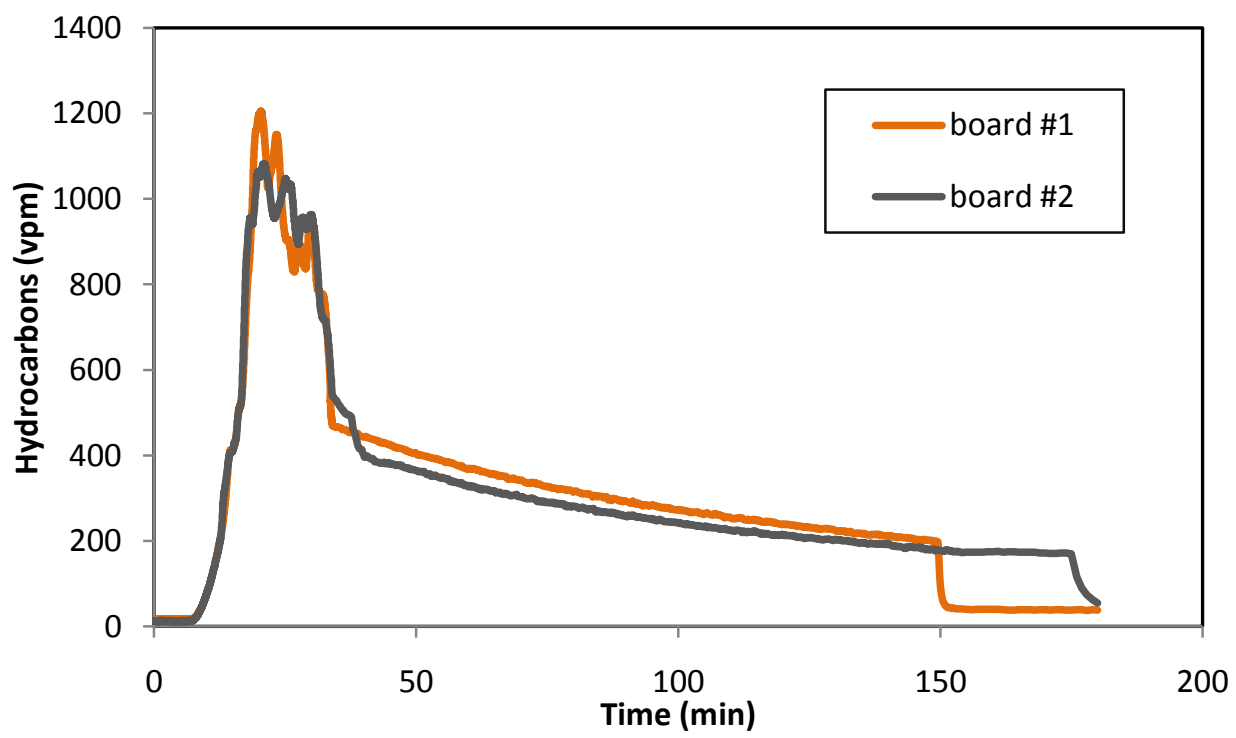

Fig. 9. CnHm hydrocarbons in the smoke. 


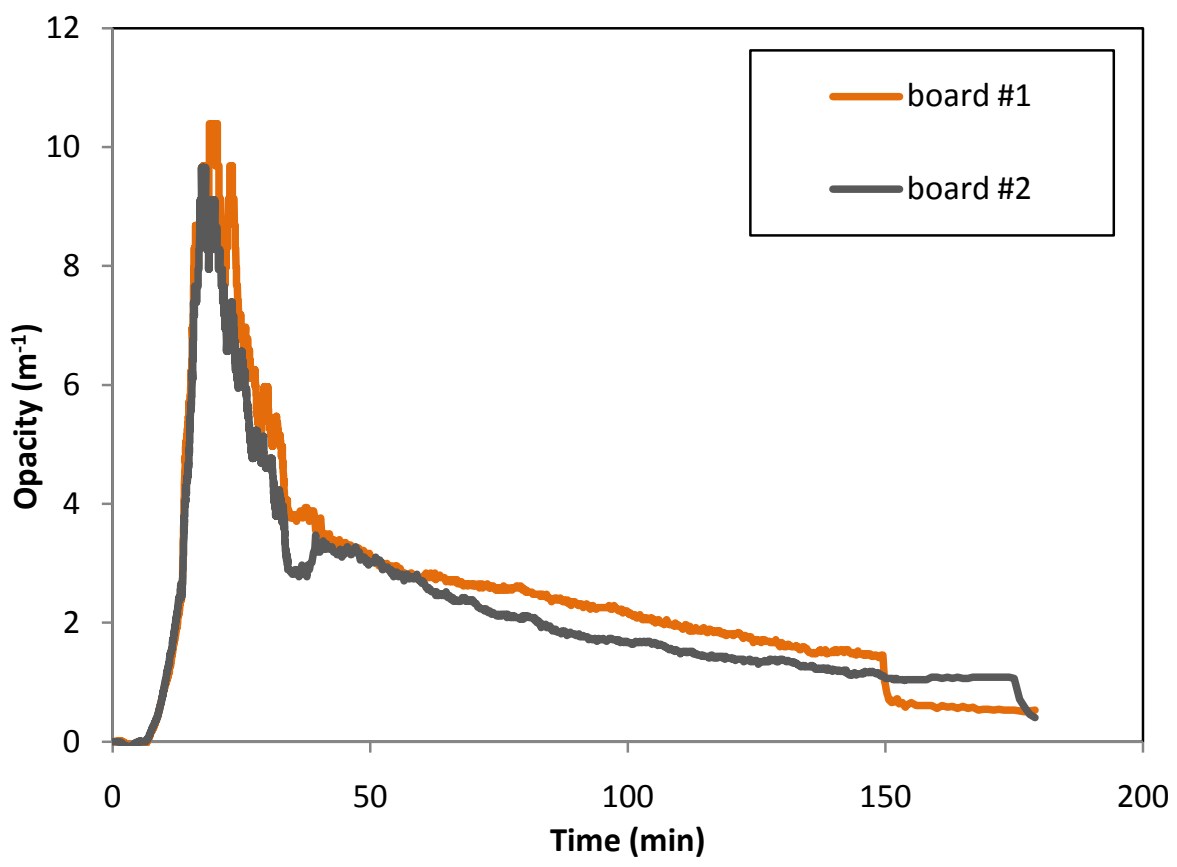

Fig. 10. Smoke opacity in the enclosure.

Although the equipment behaviour may be dependent on construction materials and equipment layout, no malfunctions occurred on either of the electronic boards during the 24 hour tests. For example, the voltage output results for board \#2 do not exhibit any values out of the functioning range (Fig. 11).

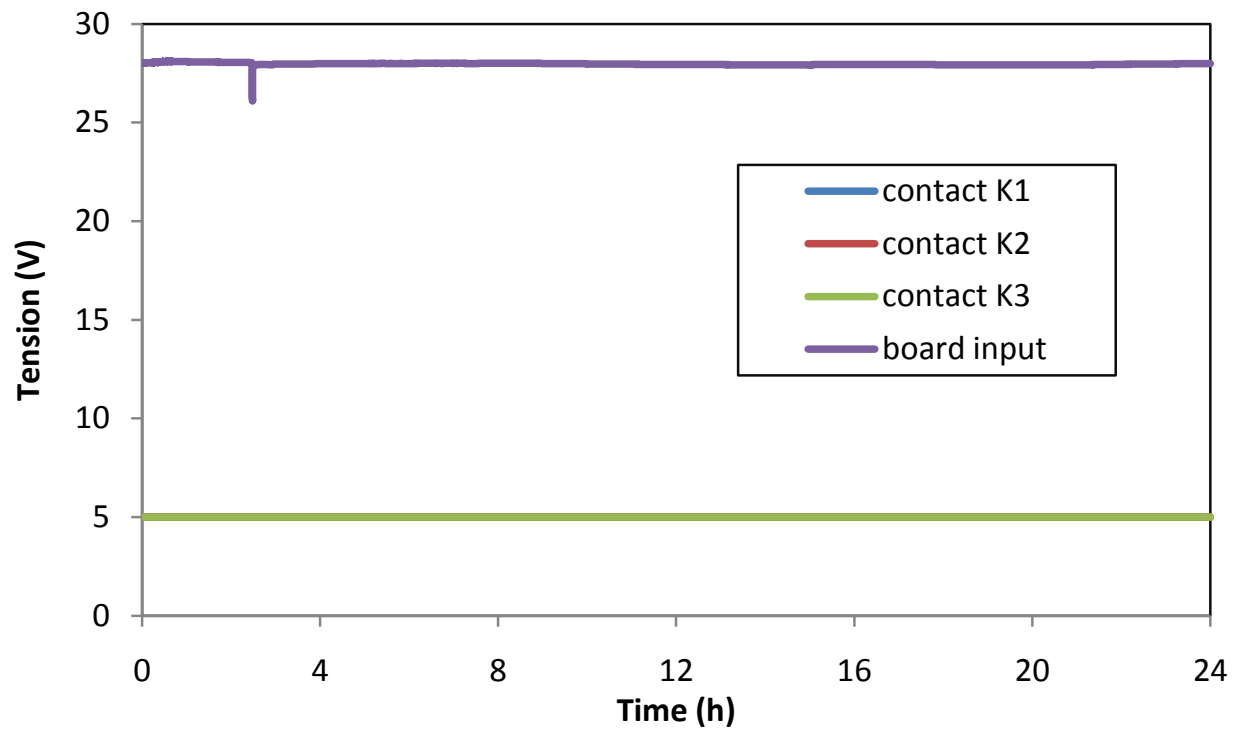

Fig. 11. Malfunction monitoring of board \#2 through voltage measurements.

Moreover, no malfunctions were detected during follow-up monitoring conducted 6 months after the smoke exposure and without any clean-up of the boards. 


\section{CONCLUSIONS}

No malfunctions were detected during board exposure to smoke at a maximum temperature of $68^{\circ} \mathrm{C}$, neither during tests after 6 months. Gases had no impact through corrosion and soot did not cause any short circuiting between the conductors.

More exhaustive tests with a wide range of NPPs electronic boards should be performed to generalise these results. And combination with different combustion fuels that produce higher soot yields could be a way to set a baseline for electronic equipment malfunction due to smoke.

\section{REFERENCES}

[1] Gay, L., Gracia, R., Wizenne, E. Thermal malfunction criteria of fire safety electrical equipment in nuclear power plants. Fire Mater., 37:151-159, doi: 10.1002/fam.2121.

[2] Mangs, J., Keski-Rahkonen, O. Acute effects of smoke from fires on performance of control electronics in NPPs. Paper No. M1804. Transactions - 16th International Conference on structural Mechanics in Reactor Technology (SMIRT), Washington DC, 12 - 17 August 2001.

[3] Tanaka, T.J., Nowlen, S.P. Effects of smoke on functional circuits, NUREG/CR-6543 SAND972544, october 1997.

[4] Korsah, K., Tanaka, T.J., Wilson, T.L. Environmental testing of an experimental digital safety channel. Technical report NUREG/CR-6406, ORNL/TM-13122, Oak Ridge National Laboratory, TN, 1996.

[5] Babrauskas, V., Ignition Handbook, FSP SPE, 2003.

[6] Drysdale, D., An Introduction to Fire Dynamics, John Wiley and Sons, Chichester, 1985, p. 146.

[7] IEC 584-2:1982. Thermocouples. Part2: Tolerances. 14p.

[8] Norm IEC 60068-1. Environmental testing. Part 1: general and guidance, Ed. 6.0, 1998.

[9] Norm IEC 60068-2-2. Environmental testing. Part 2: tests. Tests B: Dry heat, Ed. 5.0, 2007. 